\title{
CARACTERÍSTICAS, INCENTIVOS A LA PRODUCCIÓN Y DISPONIBILIDAD A PAGAR POR LECHE ECOLÓGICA EN MEDELLÍN ${ }^{*}$
}

Recibido: 02 de agosto de 2013 - Aprobado: 15 de mayo de 2014

\author{
Alejandra Calderón García** \\ David Tobón Orozco** \\ Valentina Cardona Nieto ${ }^{* * *}$ \\ Gabriel Agudelo Viana ${ }^{* * * * *}$
}

\section{RESUMEN}

En este artículo se emplea valoración contingente para evaluar mejoras hipotéticas en la calidad ambiental de la leche, y se encuentra que es posible incentivar su producción, puesto que la disponibilidad a pagar de la demanda estimada en Medellín supera los precios de mercado y los costos de oportunidad de producir leche tradicional, en cuya producción se genera contaminación y degradación de tierras, y hay problemas de control y regulación por los organismos ambientales en Colombia. La lechería ecológica puede hacer parte de los mercados verdes y ser una opción de auto-regulación ambiental al encontrarse un mercado potencial por sus distintos atributos.

\section{PALABRAS CLAVE}

Leche orgánica, incentivos, ambiente y comercio, disponibilidad a pagar, valoración contingente.

\section{CLASIFICACIÓN JEL}

D12, Q01, O30, C15, Q560.

\section{CONTENIDO}

Introducción; 1. Caracterización del sector lechero tradicional; 2. Fundamentos teóricos y experiencias de mercados de leche orgánica; 3. Diferencias de costos entre un sistema convencional y uno ecológico de producción de leche en Colombia; 4. Descripción metodología; 5. Interpretación de los resultados de las encuestas; 6. Conclusiones; Bibliografía; Entrevistas; Anexos.

Artículo de investigación financiado por el fondo de apoyo a proyectos de trabajos de grado del CODI- Universidad de Antioquia (2012) y el Grupo de Microeconomía Aplicada. Derivado del proyecto Un Modelo de Equilibrio General con externalidades y restricciones del ecosistema natural, 2011-2012, acta CIC 8733-034-11. Grupo de Microeconomía Aplicada, clasificado A2 (2014) por Colciencias.

** Economista, Universidad de Antioquia, Medellín, Colombia. Profesional Universitario, Secretaría Distrital de Desarrollo Económico, Bogotá D.C., Colombia. Correo electrónico: alejacalderon21@gmail.com.

*. Economista, Universidad de Antioquia, Medellín, Colombia. Master of Science in Economics, Universidad de Chile, Santiago, Chile. Profesor titular y coordinador Grupo de Microeconomía Aplicada, Facultad de Ciencias Económicas, Universidad de Antioquia, Medellín, Colombia. Correo electrónico: david.tobon@udea.edu.co.

**** Economista, Universidad de Antioquia, Medellín, Colombia. Gestor wealth management en Protección S.A., Medellín, Colombia. Correo electrónico: valentina.cardonan2@proteccion.com.co.

"****Estadístico, Universidad de Medellín, Medellín, Colombia. Magíster en Estadística, Universidad Nacional de Colombia, Bogotá, Colombia. Profesor titular, Facultad de Ciencias Económicas, Universidad de Antioquia, Medellín, Colombia. Correo electrónico: gagudelo127@gmail.com. 


\section{CHARACTERISTICS, PRODUCTION INSENTIVES AND THE AVAILABILITY TO PAY FOR ECOLOGICAL MILK IN MEDELLIN}

\section{ABSTRACT}

In this article, contingent valuation is used to evaluate the hypothetical improvements of the environmental quality of milk. Results show that it is possible to encourage its production given that the willingness to pay of the estimated demand for Medellin exceeds market prices and the opportunity costs of producing traditional milk, which during its production generates soil pollution and degradation, and where the Colombian environmental regulation units have problems involving regulations and control. Organic dairy can be part of green markets and an option for environmental self-regulation when finding that potential market with as many attributes as this one.

\section{KEY WORDS}

Organic milk, Incentives and trade, Willingness to pay, Contingent valuation

\section{JEL CLASSIFICATION}

D12, Q01, O30, C15, Q560.

\section{CONTENT}

Introduction; 1. Characterization of the traditional dairy sector; 2 . Theoretical guidelines and experiences of the organic milk markets; 3 . Differences between a conventional and an ecological system for milk production in Colombia; 4. Methodological description;

5. Survey result interpretation; 6. Conclusions; Bibliography; Interviews; Attachments.

\section{CARACTERÍSTICAS, INCENTIVOS À PRODUÇÃO E DISPONIBILIDADE A PAGAR POR LEITE ECOLOGICO EM MEDELLIN \\ RESUMO}

Neste artigo se emprega valoração contingente para avaliar melhoras hipotéticas na qualidade ambiental do leite, e se encontra que é possível incentivar sua produção, já que a que a disponibilidade a pagar da demanda estimada em Medellín supera os preços de mercado e os custos de oportunidade de produzir leite tradicional, em cuja produção se gera contaminação e degradação de terras, e há problemas de controle e regulação pelos organismos ambientais na Colômbia. A leitaria ecológica pode fazer parte dos mercados verdes e ser uma opção de auto regulação ambiental ao encontrarse um mercado potencial por seus diferentes atributos.

\section{PALAVRAS CHAVES}

Leite orgânico, Incentivos, Ambiente e Comércio, Disponibilidade a pagar, Valoração Contingente.

\section{CLASSIFICAÇÃO JEL}

\section{CONTEÚDO}

D12, Q01, Ou30, C15, O560.

Introdução; 1. Caracterização do setor leiteiro tradicional; 2. Fundamentos teóricos e experiências de mercados de leite orgânico; 3. Diferenças de custos entre um sistema convencional e um ecológico de produção de leite na Colômbia; 4. Descrição metodologia; 5. Interpretação dos resultados das enquetes; 6. Conclusões; Bibliografia; Entrevistas; Anexos. 


\section{INTRODUCCIÓN}

Los consumidores comienzan a preocuparse por la calidad ambiental que, sumada a la identificación de enfermedades transmitidas por alimentos industriales, reduce la confianza en los sistemas convencionales de producción y aumentan la demanda de productos ecológicos, con un crecimiento de $20 \%$ anual promedio desde principios de la década de 1980 según Espinal, Martínez y Espinoza (2005). Los mercados verdes pueden ser una oportunidad económica para productos agrícolas y pecuarios, como la leche; este es el segundo producto orgánico de consumo mundial pero en Colombia no existe el producto, y se ofrece a comercializadoras solo como producto convencional.

Desde Murgueitio (1999) se ha mencionado que en Colombia el crecimiento y las consecuencias de la ganadería sobre el ambiente deben cuestionar la manera de hacerla sostenible y productiva, y ayudar a la calidad de vida de las poblaciones rurales. Para Bradford y otros (2006) hoy se habla de producción ecológica de leche como un sistema con responsabilidad social y ambiental, y gracias a trabajos como el de la Federación Colombiana de Ganaderos (FEDEGAN) (2011) se tienen en Colombia análisis técnicos de factibilidad de hacer esta conversión, y aunque es posible la reducción de costos promedio por litro de leche ecológica producida, también se deben contabilizar tiempos de reconversión, menor número de vacas y producción promedio por cabeza en un hato ecológico.

Respecto al lado de la demanda, Xia y Zeng (2009) han encontrado disposiciones a pagar por la leche ecológica de 5-10 \% de más sobre el precio de la leche normal en Beijing, y Schröck (2011) evidencia que en Alemania es inelástica al precio en compradores habituales. Respecto al ingreso, si bien no puede asimilarse como un determinante de las preferencias en sí mismo, es un condicionante en países en desarrollo, donde se compite con la creciente oferta de sustitutos a muy bajo precio, pero con estándares ambientales malos.

En este artículo se mide la factibilidad de incentivar a los productores primarios del sector lechero colombiano para producir leche ecológica, mediante la aplicación de valoración contingente, con el fin de evaluar mejoras hipotéticas en la calidad ambiental de la leche, sobre consumidores potenciales en Medellín. Para el diseño de la encuesta se tuvo en cuenta los fundamentales de los mercados verdes. Con la información obtenida en la encuesta se estima la disponibilidad a pagar (DAP) por leche entera y deslactosada ecológica, y se compara con los costos de oportunidad que enfrentarían los productores de leche al introducir las prácticas ambientales requeridas, obteniendo un diferencial positivo, y que destaca la importancia de proveer información sobre el producto, por ejemplo sobre sus beneficios en la salud y nutricionales, sobre todo a los consumidores de leche tradicional. 
El trabajo se divide en seis partes: en la primera, se caracterizan las externalidades ambientales que genera la producción lechera, la normativa que las regula y los problemas de implementación en el sector; en la segunda, se esboza el concepto y características de los mercados verdes y la leche ecológica; luego, se muestra el análisis de costos y una comparación entre sistemas convencionales y de producción de leche ecológica en Colombia. En la cuarta, se ilustra la metodología de valoración contingente para un modelo logístico, y se comprueban e interpretan las estimaciones de la DAP. Por último, se concluye.

\section{CARACTERIZACIÓN DEL SECTOR LECHERO TRADICIONAL}

La producción de leche es una de las actividades agropecuarias que representa mayor contaminación: hay efectos sobre el suelo, el agua, el aire, y la biodiversidad —-biomasa, ecosistemas y especies_- '. Si bien es imposible hablar de una actividad productiva que no cause afectación al ambiente, sí es admisible categorizar sistemas productivos más limpios. Mahecha (2002) plantea que este es el reto al que se enfrenta la ganadería en Colombia, al esperarse que se mantengan los rendimientos productivos por unidad de área.

En el caso de los gases de efecto invernadero (GEI) emitidos por las vacas durante la digestión, aunque es improbable eliminarlos, sí es factible reducirlos, ya que dependen en mayor medida de la dieta alimenticia. Una alternativa que plantean Carmona, Bolívar y Giraldo (2005) por sus variados beneficios son los sistemas silvopastoriles (SSP), que combinan la producción ganadera con la de árboles y arbustos. Según Navia (2000), citado en YR (2011), en estos sistemas es posible limitar la emisión de metano, y obtener otros beneficios como la disminución de la degradación de la tierra, mejorar la fertilidad del suelo con incrementos en la materia orgánica y aporte de nitrógeno, control de condiciones ambientales adversas (vientos, excesiva temperatura, evaporación del recurso hídrico, etc.), sombra y alimento para animales y disponibilidad de productos comercializables como son madereros, frutos, miel, hongos y forrajes, y la protección de la propiedad con cercas vivas en lugar de metálicas y eléctricas. Los SSP se deben combinar con otras actividades para que sean considerados ecológicos; según el Observatorio de Competitividad (2002, p. 2):

\footnotetext{
1 Hay contaminación a los cauces de agua donde llega el estiércol de los animales, a la atmósfera por los gases que emiten las vacas en la digestión (metano y gas carbónico), por el uso de los químicos como fertilizantes, herbicidas, plaguicidas y fungicidas que lleva el pasto que los animales consumen y que alteran la composición físico-química de estos, los productos de limpieza usados en tanques donde se procesa y guarda la leche y que todos se escurren a los cauces, y en cuanto a las afectaciones producidas por la rotación de las tierras en los hatos, más alta en predios pequeños.
} 
La agricultura ecológica se define como un sistema de producción que utiliza insumos naturales y prácticas especiales, como la aplicación de abonos verdes, control biológico, asociación y rotación de cultivos, uso de repelentes y funguicidas a partir de plantas y minerales, entre otras y prohíbe el uso de pesticidas y fertilizantes de origen químico. En tal sentido, se denomina producto ecológico a aquel cuyo proceso de producción se acoge a dichos parámetros y ha sido certificado como tal.

Respecto a Colombia, Espinal, Martínez y Espinoza (2005, p.1) precisan que "la Resolución 0074 de 2002 del MADR establece el término de "sistema de producción ecológica", pero en general, los términos ecológico, orgánico o biológico son sinónimos". Y en cuanto a la regulación del sector la estructura institucional encargada de la política ambiental se deriva de la Ley 99 de 1993. Como parte de esta política se crearon las tasas retributivas, concebidas como instrumentos económicos, al cobrar a cada usuario por unidad de sustancia contaminante una cuantía, tal que se interiorice la decisión de contaminar y pagar, o se motive implementar soluciones tecnológicas para evitar el pago de las tasas. En el artículo 42 se menciona:

Tasas Retributivas y Compensatorias. La utilización directa o indirecta de la atmósfera, del agua y del suelo, para introducir o arrojar desechos o desperdicios agrícolas, mineros o industriales, aguas negras o servidas de cualquier origen, humos, vapores y sustancias nocivas que sean resultado de actividades antrópicas o propiciadas por el hombre, o actividades económicas o de servicio, sean o no lucrativas, se sujetará al pago de tasas retributivas por las consecuencias nocivas de las actividades expresadas.

Las tasas retributivas y sus valores están reglamentados en el Decreto 26672012.

Además, existen normas técnicas y de cantidades (comando y control) definidas en el Decreto 3930 de 2010, con lo cual se busca, en teoría, que se vierta solo el 20 $\%$ de los residuos contaminantes, y se pague sobre estos la tasa retributiva. Sin embargo, no hay reglamentos concretos de vertimientos para los productores lecheros. Por último, se tiene el Reglamento Técnico de la Producción de Leche (Decreto 616 de 2006), asociado al cumplimiento de parámetros técnicos que afectan la calidad de la leche pero no el ambiente.

Para precisar el tipo de regulación ambiental en Colombia, según Tobón y Vasco (2011, p. 10):

Los instrumentos utilizados para regular externalidades se pueden agrupar en comando y control cuando se trata de restricciones a las emisiones —estándares de desempeño—y restricciones tecnológicas — estándares de diseño—, o de incentivos, como son impuestos, subsidios, transacción de derechos o permisos, y depósitos reembolsables. Su efectividad depende de la posibilidad de medir todos los daños marginales indirectos, bajos costos de transacción de mercado, la posibilidad de definición y protección de derechos de propiedad, la facilidad de implementabilidad, regulación y vigilancia, el apetito fiscal de los estados, y las posibilidades técnicas y 
los costos de usar tecnologías de mitigación. En cuanto a los productos verdes y la responsabilidad social ambiental se pretende explotar la sensibilidad de la demanda por estos productos diferenciados.

El problema concreto en Colombia es que, si bien existe una completa normativa ambiental para este sector, como sostienen Galarza (2009) y la Corporación Autónoma Regional del Centro de Antioquia (Corantioquia), la Universidad Nacional y la Universidad de Antioquia (2012), hay problemas de implementación y cumplimiento, es decir, hay resistencia, falta de formalización y evasión, por lo que no se hace inversión en descontaminación ni se pagan los costos sociales que se generan; esto hace referencia a la ineficiencia de la administración y de la gestión de las autoridades ambientales, y la manera como se calcula la tasa retributiva. Ahora bien, el punto de partida de este trabajo es que, aunque la regulación no funcione y haya fallas para hacer cumplir la ley, puede existir una porción de mercado por productos verdes que incentive a que en el sector se cumpla con mejores estándares ambientales.

\section{FUNDAMENTOS TEÓRICOS Y EXPERIENCIAS DE MERCADOS DE LECHE ORGÁNICA}

Para el Ministerio de Ambiente y Desarrollo Sostenible (MADS) (2002) los mercados verdes se definen como aquellos en los cuales los productos son sustentables, y tienen como señal una certificación ambiental: vienen de procesos de producción amigables con el ambiente y con impacto social positivo. Siguiendo a Borregaard y otros (2003) el inicio de este tipo de mercado, el cual comenzó como comercio justo o de impacto social positivo, data de la década de 1970, mientras el comercio con criterios ambientales se promovió una década después.

Los productos verdes tienen criterios de clasificación más estrictos, y se denominan así según la normativa que tenga cada país o la respectiva industria. Según el MADS (2005) para que un producto colombiano tenga sello verde debe cumplir con buen uso de los recursos renovables, utilizar materias primas que no sean nocivas para el ambiente, procesos de producción que ahorren recursos y energía y posibiliten reciclar, reutilizar, utilizar materiales biodegradables, entre otros. También importan su lugar de origen, frescura de almacenamiento, empaquetamiento, transporte y favorecimiento de empleo y comercio justos. El MADS (2005) aclara que en Colombia el Ministerio de Ambiente, Vivienda y Desarrollo Territorial (MAVDT) y el Ministerio de Comercio, Industria y Turismo (MCIT) crearon el Sello Ambiental Colombiano (SAC) y reglamentaron su uso mediante la Resolución 1555 de 2005.

Ahora bien, estos productos no tienen gran cuota de mercado debido a que se espera que tengan costos más altos comparados con uno convencional. Según el Ministerio de Agricultura y Desarrollo Rural (MADR) (2007), tomando como referencia 
el sistema de información del Center for the Promotion of Imports from Developing Countries (CBI), los productos orgánicos pueden presentar un sobrecosto entre $15 \mathrm{y}$ $40 \%$, asociado a los costos de producción, certificación y comercialización. Además, en América Latina existe un crecimiento importante: la oferta está representada por Argentina, 68 \%; Uruguay, 14 \%; Brasil, 6 \%; Chile, 6 \%; Perú, 2 \%; Colombia, 2 \%; Paraguay, $1 \%$, y Ecuador, $1 \%$. El consumo global se concentra en frutos y hortalizas (30\%), lácteos (15\%), cereales (15\%) y carne (10\%). Y los mercados externos de mayor importancia son Estados Unidos y Europa.

De acuerdo con Van Hoof, Monroy y Saer (2008) si el sello ambiental está en un producto y este es reconocido como tal, se pueden alterar las motivaciones de los consumidores. Para Toro y Madrid (2012) este valor agregado es una alternativa de mayores ingresos, puesto que cada vez hay mayor demanda de productos que provengan de buenas prácticas ambientales y sociales, y por los que están dispuestos a pagar un precio más alto.

Para Brécard y otros (2009), Frey (1999), entre los fundamentos microeconómicos que afectan la decisión de compra de este tipo de bienes sobresalen las motivaciones intrínsecas (obligación moral o altruista con el ambiente, sanciones sociales, mejoras en salud y la preocupación por la calidad del producto en lugar de la cantidad), aunque la aplicación de regulaciones y subsidios puede trasladar la iniciativa de los consumidores al Estado. Tanner y Wolfing (2003) precisan que hay barreras personales asociadas al conocimiento del producto, actitudes específicas (sobre otras características del producto), barreras percibidas y contextuales (convicción que la decisión de compra hace parte del engranaje que impulsa los mercados verdes) o si existen puntos de venta accesibles. Las percepciones sobre la calidad del producto dependen de las características socioeconómicas de los consumidores.

Sin embargo, puesto que las cualidades de los productos verdes no son observables se presenta un problema de selección adversa que es resuelto mediante el etiquetamiento del producto (sello verde), pero puede implicar un mayor precio; Mahenc (2008) indica que cuando los consumidores no pueden identificar la calidad ambiental de un producto el precio debe distorsionarse hacia arriba para distinguir su calidad.

Un producto verde también se puede clasificar como un bien público impuro, puesto que para consolidarse como producto quienes tienen preferencias por él esperan a que otros lo compren y faciliten el ciclo de vida del producto, para que luego sea asequible a un menor precio, lo cual implica que la provisión que hace el mercado es ineficiente. 
Respecto al ingreso, si bien no es un determinante de las preferencias en sí mismo, es un limitante a la demanda; de hecho, la DAP (gusto por la calidad) que se quiere estimar podría interpretarse como la sustitución marginal entre ingreso y calidad. Por tanto, los consumidores pueden preferir productos verdes pero demandan estándares ambientales menores por sus restricciones de ingresos. El fenómeno se refuerza por la creciente oferta de sustitutos a muy bajo precio, pero con estándares ambientales malos.

\subsection{Leche orgánica}

De manera simple, según Toro y Madrid (2012) las vacas en producción orgánica reciben una alimentación más saludable; en lugar de cultivos que utilizan agroquímicos y transgénicos, se manejan mejor los residuos contaminantes, y los suelos se presionan menos. Mena (2009) agrega que la leche ecológica contiene menos grasas saturadas, y más omega 3 y ácido linoleico que la tradicional.

Xia y Zheng (2009) muestran que los consumidores deciden comprar leche orgánica por la utilidad en salud, además de la percepción sobre riesgos y peligros transmitidos por los alimentos tradicionales, lo cual depende de factores socioeconómicos. Aunque el cuidado por el ambiente influye en el consumo de productos ecológicos es mucho más significativo el aporte a la salud. En Beijing la disposición a pagar por esta leche se concentra en un rango de 5\%-10 \% de más sobre la leche normal. Pero allí la mayoría de los hogares están familiarizados con los alimentos verdes, tienen actitudes positivas hacia ellos, y le dan gran importancia a la marca ${ }^{2}$. La edad, la percepción que tienen de los alimentos verdes y el acceso a la información son los principales factores que influyen en la disponibilidad a pagar. Para Mei, Ling y Hooi (2012) el conocimiento del ambiente, la actitud hacia este, la iniciativa gubernamental y la presión de los grupos de interés influyen sobre la intención de compra del consumidor de productos verdes en Malasia. Y si bien es importante la eco-etiqueta, esta no tiene mayor relevancia en la elección de consumo de estos productos.

Schröck (2011) menciona que la leche orgánica representa el 14 \% de las ventas en el mercado de alimentos orgánicos alemán. La demanda por leche convencional es elástica, mientras que la de leche orgánica es más inelástica, sobre todo por parte de compradores habituales comprometidos (lado izquierdo del gráfico 1). También las características sociodemográficas influyen en la decisión de compra; en los hogares

\footnotetext{
${ }^{2}$ La emisión de etiquetas falsas hace que los consumidores pierdan confianza en los productos verdes lo cual implica que confíen más en las grandes marcas, porque creen que se controlan con más facilidad por los organismos certificadores.
} 
donde hay un solo niño hay mayor probabilidad de que compren leche orgánica. Por último, el aumento en la preferencia por la alimentación saludable ha contribuido al crecimiento de la demanda de leche orgánica.

Gráfico 1. Comparación de la elasticidad precio por leche orgánica y convencional en Alemania, 2005-2008

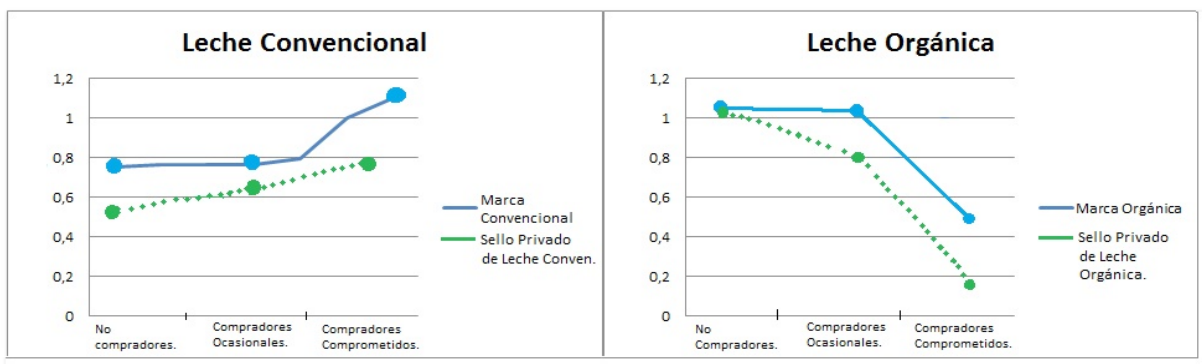

Fuente: tomado de Schröck (2011)

\section{DIFERENCIAS DE COSTOS ENTRE UN SISTEMA CONVENCIONAL Y UNO ECOLÓGICO DE PRODUCCIÓN DE LECHE EN COLOMBIA}

En Colombia, la leche orgánica no se vende como tal y no se daría una conversión a sistemas ecológicos con facilidad, pero siguiendo a Márquez (2012; 2011) sí es posible mantener sistemas agroecológicos en el trópico, aunque, como se ha mencionado, se requiera reducir el número de vacas y la producción de leche por vaca en comparación con un sistema convencional. No obstante, ella sugiere que la principal restricción es cultural, al ser los productores con aversión a cambiar sus sistemas de producción, pues se han adaptado a la lechería tradicional en la cual se facilita su venta a los comercializadores-pasteurizadores en lugar de tenerse que arriesgar a vender en el mercado, como ocurre con otros productos agropecuarios. FEDEGAN (2012) menciona el caso de la hacienda San José en Pasto, la cual ha aumentado la productividad incluso con el sistema ecológico en cuanto a rotación de praderas, producción de leche y capacidad de carga, y reducción en el uso de medicamentos, productos químicos de limpieza, pesticidas, herbicidas, etc.

Sin embargo, Espinal, Martínez y Espinoza (2005), Pérez y Álvarez (2008) afirman que si bien en la producción de leche ecológica los costos pueden ser menores debido a la no utilización de medicamentos, fertilizantes, fungicidas, insecticidas y venenos químicos, también es cierto que estos pueden aumentar al requerir más vacas por hectárea o mayores precios por litro de leche, costos de certificación y mano de obra en mayor cuantía y especializada. Además, los medicamentos deben ser de origen biológico, a no ser que la normativa obligue a usar determinados 
medicamentos de origen químico, y se prohíbe el uso de sustancias artificiales para estimular el crecimiento y la reproducción del ganado, lo que puede aumentar los costos en comparación con los sistemas convencionales.

Además, se consideró la opinión de algunos productores de leche en San Pedro de los Milagros (Antioquia), sobre sus métodos de producción y qué tan dispuestos estarían a realizar la reconversión de la finca y a ofrecer leche ecológica. Estos se muestran interesados, aunque en general poco conocen sobre leche ecológica a pesar de haber tenido experiencias con cultivos orgánicos, que prefieren por más baratos y porque mejoran las pasturas de los potreros. Velásquez (2012), quien le vende su producción a la pasteurizadora Colanta, comenta que es muy complicada la producción de leche ecológica en Antioquia por tratarse del trópico y por ser más propensos a los parásitos, y además, porque la prohibición de químicos facilita que los animales mueran. Pero Martínez (2012) y Ospina (2012) son más positivos al reconocer que igual cuentan en el momento con poca asistencia técnica por parte de Colanta, quien les compra toda la leche.

Ahora bien, para hacer la comparación de costos se tomó como referencia el análisis de EMPRENDEGAN (2010) sobre la finca El Recreo en Abejorral (Antioquia), productora de leche convencional, similar en extensión a la hacienda San José (30 ha), ecológica, con menores costos operativos, pues el costo de producir un litro de leche entera es $\$ 551,51$, a precios de 2013, mientras en El Recreo es de $\$ 638,74$.

Sin embargo, en la conversión aparecen otros costos al necesitarse de un período de preparación de terrenos y animales, cercano a seis meses para la producción de leche, según el MADR (2006). Además, si no se dispone de animales ecológicos certificados podrán introducirse animales no ecológicos pero menores de seis meses; si en la tierra se han usado químicos sintéticos, es necesaria una reconversión de dos años en lugar de seis meses. Al hacerse un cálculo conservador, como indica Márquez (2012), el número de vacas se podría reducir a la mitad, al requerirse cercas naturales y espacios para siembra de plantas y leguminosas para el control de plagas y la alimentación balanceadas de las vacas que restan el área de pastos, y se merma la producción promedio de litros de leche por vaca de 16 (promedio nacional) a $14^{3}$.

Obsérvese de la tabla 1, que el productor de leche ecológica (Ecol) encara menores costos operativos $(\mathbf{C} / \mathbf{L})$, pero debe sumar los de conversión $(\mathbf{C} * / \mathbf{L})$. Los nuevos costos que lo hacen indiferente entre producir leche entera convencional y entera ecológica son $\mathbf{C}^{*} / \mathbf{L} \mathbf{m a x}$, y se obtienen si se supone que el precio al consumidor final es igual a la DAP encontrada en este estudio (\$3112), pero debe compensar el

\footnotetext{
3 Para el precio de la leche se tiene una referencia definida por el MADR (Resolución 017 de 2012), y el Decreto 616 de 2006 reconoce bonificaciones o reducciones al precio de acuerdo con las calidades higiénicas y composicional de la leche.
} 
déficit diario de ingresos de $\$ 1.018 .029,6$, o sea, $\$ 1001,8$ por litro. Por otro lado, los costos de conversión mínimos requeridos $(\$ 87,23)$ se calculan como el diferencial entre los costos operativos de leche convencional y de leche ecológica observados, es decir, como un costo de oportunidad equivalente asociado a las adecuaciones que deben hacerse y compensan la reducción de costos operativos que se obtiene al producir leche ecológica en la finca San José.

Tabla 1: Costos leche entera finca San José (Eco) frente a El Recreo (Conv.)

\begin{tabular}{|c|c|c|c|c|c|c|c|c|c|c|c|c|c|c|}
\hline & $\mathrm{Ha}$ & V & $L / V$ & & Total & $\mathrm{C} / \mathrm{L}$ & $\mathrm{C}^{*} / \mathrm{L} \mathrm{C}^{*}$ & /Lmax & CT./L & $\mathrm{T}$ & P. Prod. Ingr & Utilidac & Déficit & Sobreprecio \\
\hline Eco & 30 & 60 & & 840 & 551,51 & 87,23 & & 638,74 & 536541,6 & 870 & 730800 & 194258,4 & 249760,8 & 297,33 \\
\hline Eco 1 & 30 & 60 & 14 & 840 & 551,51 & & 1001,83 & 1553,34 & 1304810,4 & 870 & 730800 & $-574010,4$ & 1018029,6 & 1211,94 \\
\hline Conv & 30 & 120 & 16 & 1920 & 638,74 & 0 & & 638,74 & 1226380,8 & 870 & 1670400 & 444019,2 & & \\
\hline
\end{tabular}

Fuente: elaboración propia

De la tabla 2 se tiene que el máximo precio que debe recibir el productor por litro de leche ecológica es de $\$ 2081,94$, en comparación con los $\$ 870$ actuales que obtiene del pasteurizador, para compensar el déficit obtenido con $\mathbf{C} * \mathbf{L} \mathbf{m a x}$. Ahora bien, es exagerado tener este costo de oportunidad de referencia; por ejemplo, si se asume un período de conversión de ciento veinte días (seis meses), y se mantiene la producción diaria en 840 litros (\$730.800 diarios), se dejarían de producir 841.542 litros y con ellos los costos de oportunidad serían de $\$ 100.985 .040$ para todo el periodo, y $\$ 841.542$ por día, lo cual también es excesivo para una finca de 30 ha. Por tanto, hay señales para indicarle al productor que los costos de oportunidad por litro producido no pueden ser tan altos, y que para la DAP estimada hay incentivos reales para producir leche ecológica.

Tabla 2: Precio producción requerido leche entera ecológica

\begin{tabular}{|c|c|c|c|c|}
\hline & \multicolumn{3}{|c|}{ P. Prod. Requerido Comercialización Precio F } & DAP* \\
\hline Eco & 1167,33 & 1030 & 2197,33 & 3111,94 \\
\hline Eco 1 & 2081,94 & 1030 & 3111,94 & \\
\hline Conv & 870 & 1030 & 1900 & \\
\hline
\end{tabular}

Comerc: Costo comercialización imputado a Colanta. Precio F: Precio final. DAP:Disponibilidad a pagar estimada.

Fuente: elaboración propia

\section{DESCRIPCIÓN METODOLOGÍA}

La valoración contingente (VC) facilita simular un mercado hipotético para la leche ecológica, en el cual los consumidores pueden expresar su valoración por los atributos del bien y revelar su disponibilidad a pagar. El cambio en el bienestar se 
estima al comparar dos funciones de utilidad indirecta: se obtiene un valor ex ante aún si el cambio no ha ocurrido, y se encuentra tanto la máxima disponibilidad a pagar (Mdap) como la mínima disposición a ser compensado (Mdac) si se trata de medir el impacto de un cambio negativo; aquí se emplea la Mdap.

El punto de partida es suponer una función U que represente las preferencias del consumidor, de forma general:

$$
U=U(J P, Y, S)
$$

Sea:

J: Indica la existencia del bien o servicio. J=1 si el consumidor está Dispuesto a Pagar (DAP) un monto determinado por su provisión. Si J=0 no está DAP.

P: Precio de los bienes.

Y: Ingreso.

S: Características socioeconómicas.

Pero como no se conoce la función $U$ directa entonces se iguala a su valor esperado, la función indirecta $\mathrm{V}$ representa la parte que se puede conocer de la utilidad, así:

$$
U=U(J P, Y, S)=V(J P, Y, S)+\varepsilon
$$

Donde $\varepsilon$ es el término de error, tal que $E(\varepsilon)=0$

La no provisión del bien genera una utilidad $V_{0}=V_{0}(0, P, Y, S)+\varepsilon_{0}$. Y si el mercado provee el bien: $V_{1}=V_{1}(1, P, Y, S)+\varepsilon_{1}$.

Se espera que la provisión genere mayor utilidad, en este caso, bajo el supuesto de que la leche ecológica proporciona mayor bienestar; por tanto:

$$
V_{1}=V_{1}(1, P, Y, S)+\varepsilon_{1} \geq V_{0}=V_{0}(0, P, Y, S)+\varepsilon_{0}
$$

Si lo anterior se cumple, se provee el bien; de lo contrario el consumidor no estará dispuesto a pagar un monto adicional.

Sea $\mathbf{A}$ la cantidad de dinero que él estaría dispuesto a ceder para obtener los beneficios de la leche ecológica. Esta disponibilidad está limitada por su capacidad de pago, es decir: $0 \leq D A P \leq Y$. Si se define $\mathbf{C}$ como su máxima disposición a pagar: $C=C(J P, Y, S)$, el consumidor estará dispuesto a pagar por la leche ecológica 
siempre y cuando A sea inferior a $\mathbf{C}$, y "No" en caso contrario; solo si $\mathbf{A}<\mathbf{C}$, el consumidor acepta pagar A para mantener la provisión del bien, y su utilidad será entonces: $V_{1}=V_{1}(1, P, Y-A, S)+\varepsilon_{1}$.

Entonces:

$$
V_{1}=V_{1}(1, P, Y-A, S)+\varepsilon_{1} \geq V_{0}(0, P, Y, S)+\varepsilon_{0}
$$

Se supone que los errores son variables aleatorias, independientes e igual distribuidas.

Al despejar de (4) se tiene que:

$$
V_{1}(1, P, Y-A, S)-V_{0}(0, P, Y, S) \geq \eta
$$

De donde $\eta=\varepsilon_{0}-\varepsilon_{1}$ equivale a los errores de estimación y $V_{1}-V_{0}=\Delta V$, entonces:

$$
\Delta \mathrm{V} \geq \eta
$$

Por tanto: $\operatorname{Pr}$ (el entrevistado responda que "Sí" está DAP $)=\operatorname{Pr}(\Delta \mathrm{V} \geq \eta)=\mathrm{F}(\Delta \mathrm{V})$ F es la función de probabilidad acumulada, se supone que distribuye normal (Probit) o logística (Logit). De acuerdo con Fernández y Pérez (2005, p. 5), en este trabajo se usará Logit por estar más acorde con la distribución de los residuales y tener unas colas más pesadas; además, sirve cuando se necesita predecir un resultado binario (en este caso, de la preferencia o no por la leche ecológica) y se asume que existen varios factores que pueden incidir sobre tal resultado. La función logística relaciona la variable dependiente $Y_{t}$ con las variables independientes $X_{1}, X_{2}, \ldots, X_{k}$ a través de la siguiente ecuación:

$$
\mathrm{Y}_{\mathrm{t}}=\frac{1}{1+\mathrm{e}^{-\mathrm{z}}}+\mu_{\mathrm{t}}
$$

$Y_{\mathrm{t}}$ puede tomar valores de cero y uno, $\mathrm{z}_{\mathrm{t}}$ es el Scoring logístico, $\mathbf{Z}=\beta_{0}+\beta_{1} \mathbf{X}_{1}+\ldots+\beta_{\mathrm{k}} \mathbf{X}_{\mathrm{k}^{\prime}}$ o de forma matricial: $\mathbf{Z}=\beta \mathbf{X}, \mathrm{y} \mu_{\mathrm{t}}$ es una variable aleatoria que distribuye $\mathrm{N}\left(0, \sigma^{2}\right)$.

\section{INTERPRETACIÓN DE LOS RESULTADOS DE LAS ENCUESTAS}

La VC se fundamenta en información recolectada por encuestas a grupos de usuarios con representatividad estadística, e indaga por la disposición a pagar 
(DAP) por el bien ofrecido, en este caso leche ecológica. No obstante, Osorio y Correa (2004) listan los sesgos más comunes en estos estudios: de información (debido a la poca información que puede tener el encuestado respecto al efecto que tendrá su DAP sobre la decisión derivada del estudio), punto de partida (surge de la pregunta dicotómica de doble límite, ya que el valor inicial que se toma como base puede determinar la respuesta final obtenida), del entrevistador (presión que puede ser voluntaria o involuntaria) y estratégico (cuando los entrevistados buscan modificar la decisión de pago, al reportar valores de DAP diferentes a su utilidad marginal real).

Con el fin de reducir al máximo estos sesgos y corregir errores en los cuestionarios se realizaron 25 pruebas piloto. En estas se pudieron identificar dos tipos de leche con mayores consumos: leche entera y deslactosada, por ello la pregunta sobre la DAP depende del tipo de leche consumida. Asimismo, se efectuaron cuatro tipos de encuestas, que preguntan por diferentes disposiciones de pago de forma aleatoria con el fin de reducir los sesgos de la puja inicial. A los encuestados se les describieron las características de la leche ecológica, y tras un juego iterativo que busca un simple Sí/No, se realizó una pregunta abierta sobre la máxima DAP por un litro de leche ecológica

La población estuvo constituida los hogares de estratificación socioeconómica 4, 5 y 6 de Medellín. Según la Encuesta de Calidad de Vida (2011), hay 78514 hogares de estrato 4 (medio), 58132 de estrato 5 (medio-alto) y 31.190 de estrato 6 (alto), para un total de 167836 hogares. La fórmula empleada para definir el tamaño muestral de una población finita es:

$$
\mathrm{n}=\frac{\mathrm{n}_{0}}{1+\mathrm{n}_{0} / N}
$$

\footnotetext{
${ }^{4}$ Se pregunta así: ¿Usted estaría dispuesto a pagar "\$A" para que exista el bien en el mercado?, y se sigue un juego iterativo. El precio inicial se aumenta para contrastar si el encuestado seguiría de acuerdo en pagarlo y así hasta que declare que "No" está dispuesto a pagar el aumento adicional. Se sugiere que el segundo precio esté comprendido en un intervalo más largo respecto al anterior, para que el encuestado llegue a responder "No". La última valoración aceptada es la máxima DAP. Esto hace que el modelo sea dicotómico de doble límite.
} 
$\mathbf{N}$ es el tamaño del universo y $\mathbf{n}_{0}=\mathbf{p}(1-\mathbf{p})\left|\frac{\not \mathbf{z} 1-\alpha / 2}{d}\right|^{2}$, $\mathbf{p}$ la probabilidad de ocurrencia y d, el error máximo de estimación. Este cálculo arroja una muestra de 304 para un p-valor del 0,5\%, un nivel de significancia de $97 \%$ y un margen de error de 6,26\%, que es la que se aplicó 5 .

Las pruebas de adecuación del primer modelo, que estima la probabilidad de consumir leche ecológica entera indican que es función de las variables independientes significativas ${ }^{6}$ :

P.4 ¿Ha oído hablar de productos ecológicos? ${ }^{7}$

P.10 ¿Cuántos litros de leche se consumen en el hogar a la semana?

P.11 ¿Qué tipo de leche se consume en su hogar en mayor proporción??

P.14.TOTAL.MAX ¿Cuánto es su máxima DAP por un litro de leche ecológica?

P.16 ¿Cuál es la principal razón por la que le interesa la leche ecológica?10

SDP.9 ¿Cuánto pueden sumar sus gastos mensuales?"1

Al relacionar la variable Máxima DAP con cada una de las variables significativas, se obtiene que de los hogares encuestados el $81,3 \%$ ha oído hablar de productos ecológicos, el 42,8 \% consumen entre uno y cinco litros de leche a la semana. Además, un 41,4 \% consume en mayor proporción leche entera, 35,5 \% consume leche deslactosada y el restante otro tipo de leche (descremada, semideslactosa, semidescremada, mixta); de hecho quienes consumen leche deslactosada están dispuestos a pagar un precio más alto $(\$ 3720,37)$ por la leche ecológica en comparación con los consumidores de leche entera $(\$ 2921,43)$. Asimismo, la razón principal por la que les interesa la leche ecológica es por salud (62,8 \%) y son los que están

5 Las tablas y gráficos siguientes están basados en la encuesta aplicada, y el modelo econométrico se trabajó en el software SPSS. El lector interesado en la base de datos puede solicitarla a la dirección electrónica: david.tobon@udea.edu.co.

6 Las otras variables no significativas son: Realización de actividades de cuidado y conservación del ambiente, consumo de productos con sello ecológico, edad, sexo, grado de educación, ocupación e ingresos mensuales aproximados del hogar.

7 Las variables P.10, P.11, P.16 y SDP. 9 fueron categorizadas para facilitar su análisis, mientras que P.4 es dicotómica y SDP 9 se tomó como pregunta abierta.

8 Esta variable se ubica en tres intervalos, debido a los pocos casos que se presentaban en algunas opciones, así: 1. Entre 1-5 litros de leche a la semana. 2. Entre 6-10 litros. 3. Más de 11 litros.

9 Las opciones en esta pregunta se agruparon así: 1. Hogares que consumen leche entera en mayor proporción, 2. Consumen otros tipos de leche, 3. Consumen leche deslactosada.

${ }^{10}$ Las respuestas se agruparon en: 1. Si al hogar le interesa la leche ecológica por salud, 2. Por sostenibilidad ambiental, 3. Propiedades nutricionales, 4. Otras razones.

${ }^{11}$ El gasto está representado en dos categorías: 1. Menos de 2'000.000, 2. Más de 2'000.000. 
dispuestos a pagar más (\$3432,46 pesos) (tabla 3). Para el 21,7 \% la razón principal es por sostenibilidad ambiental, el 8,2 \% por propiedades nutricionales y el 7,2 \% por otras razones (le gustan los cambios o tiene los recursos para pagar un sobreprecio por el producto).

Tabla 3: DAP y razón principal para consumir leche ecológica entera

\begin{tabular}{|l|c|c|c|c|}
\hline $\begin{array}{l}\text { P. 16.a ¿Cuál es la razón principal por } \\
\text { la que le interesa la leche ecológica? }\end{array}$ & Media & N & Desv. típ. & $\begin{array}{c}\text { \% del total } \\
\text { deN }\end{array}$ \\
\hline 1. Salud & 3432,46 & 191,00 & 1236,16 & 0,63 \\
\hline 2. Sostenibilidad ambiental & 3319,70 & 66,00 & 1439,90 & 0,22 \\
\hline 3. Propiedades nutricionales & 3384,00 & 25,00 & 836,00 & 0,08 \\
\hline 4. Otros & 1590,91 & 22,00 & 1173,48 & 0,07 \\
\hline Total & 3270,72 & 304,00 & 1332,94 & 1,00 \\
\hline
\end{tabular}

Fuente: elaboración propia

Tabla 4: Variables modelo de regresión leche ecológica entera (1)

\begin{tabular}{|c|c|c|c|c|c|c|c|}
\hline & & B & E.T. & Waid & $g l$ & Sig. & $\operatorname{Exp}(\mathrm{B})$ \\
\hline \multirow{13}{*}{$\begin{array}{l}\text { Paso } \\
\text { la }\end{array}$} & P_4(li: Ha oído hablar de productos ecológicos & 4,05 & 1,50 & 7,24 & 1,00 & 0,01 & 57,15 \\
\hline & $\begin{array}{l}P_{-} 10 \text { _a: Litros de leche que se consumen en el hogar a } \\
\text { la semana }\end{array}$ & & & 9,57 & 2,00 & 0,01 & \\
\hline & $\mathrm{P}_{-} 10_{-} \mathrm{a}(\mathrm{l})$ : Entre lyS litros & 1,23 & 0,90 & 1,85 & 1,00 & 0,17 & 3,42 \\
\hline & $P_{-} 10_{-} a(2):$ Entre 6 y 10 litros & 3,93 & 1,28 & 9,46 & 1,00 & 0,00 & 51,05 \\
\hline & $\begin{array}{l}\mathrm{P}_{-} \mathrm{Il}_{-} \text {a: Tipo de leche que se consume con mayor pro- } \\
\text { porción }\end{array}$ & & & 29,03 & 2,00 & 0,00 & \\
\hline & $\mathrm{P}_{-}{ }_{\mathrm{l}}{ }_{-} \mathrm{a}(\mathrm{l})$ : Leche Entera & 11,66 & 2,26 & 26,54 & 1,00 & 0,00 & 116041,04 \\
\hline & $\mathrm{P}_{-}{ }{ }_{-} \mathrm{a}(3)$ : Otro tipo de leche & 11,89 & 2,28 & 27,28 & 1,00 & 0,00 & 145248,79 \\
\hline & $\begin{array}{l}\text { P_ }{ }^{14} \text { _- Total__ Máx: Máxima disposición a pagar por un litro } \\
\text { de leche entera ecológica }\end{array}$ & 0,00 & 0,00 & 8,98 & 1,00 & 0,00 & 1,00 \\
\hline & $\begin{array}{l}\text { P_16a: Razón principal por la cual consumiría leche } \\
\text { ecológica }\end{array}$ & & & 12,35 & 3,00 & 0,01 & \\
\hline & $P_{\text {_ }}$ 16a(l): Salud & 5,24 & 1,56 & 11,28 & 1,00 & 0,00 & 188,91 \\
\hline & $P_{-}$16a(2): Sostenibilidad ambiental & 4,37 & 1,89 & 5,33 & 1,00 & 0,02 & 78,65 \\
\hline & P_ 16a(3): Propiedades nutricionales & 6,75 & 2,11 & 10,21 & 1,00 & 0,00 & 853,68 \\
\hline & SDP_ 9 _ b(l): Gastos aproximados mensuales del hogar & 1,80 & 0,98 & 3,43 & 1,00 & 0,06 & 6,08 \\
\hline & Constante & $-17,44$ & 4,00 & 19,01 & 1,00 & 0,00 & 0,00 \\
\hline
\end{tabular}

a. Variable(s) introducida(s) en el paso 1: $\mathrm{P}_{-} 4, \mathrm{P}_{-} 10_{-} \mathrm{a}_{1} \mathrm{P}_{-} \mathrm{Il}_{-} \mathrm{a}, \mathrm{P}{ }_{-} 14_{-}$Total Máx, $\mathrm{P}_{-} 16 \mathrm{~b}, \mathrm{SDP}{ }_{-}{ }_{-} \mathrm{b}$. B es el coeficiente estimado, E.T. el error estándar y gl los grados de libertad.

Fuente: elaboración propia 
La tabla 4 muestra los estimados y las pruebas individuales, es decir, $\mathrm{H}_{0}: \beta=0$ vs. $\mathrm{H}_{1}: \beta \neq 0$. El test de Wald comprueba la significancia de todos los parámetros con un $\mathrm{p}$-valor $<0,05$, excepto SDP ${ }_{-}{ }{ }_{-} \mathrm{b}$ (1) (gasto) que puede tolerarse con un p-valor $<0,07$. Los coeficientes B son positivos, por lo que la probabilidad de que los hogares estén dispuestos a pagar por leche entera ecológica incrementa ${ }^{12}$.

En el grupo de consumidores que no ha oído hablar de productos ecológicos, los que han oído hablar ( $\left.\mathrm{P}_{\text {_ }} 4(1)\right)$ tienen 57,15 veces más posibilidades de consumir leche ecológica. En el grupo de quienes no consumen leche, los que consumen entre 1 y 5 litros tienen 3,42 veces más probabilidades de consumir leche ecológica. De manera análoga para los que consumen entre 6 y 10 litros, estos tienen 51,05 veces más probabilidades de consumir leche ecológica, a medida que más se consume leche en la semana más probabilidad se tiene de consumir leche ecológica. Y para el grupo de consumidores por el tipo de leche que se consume con mayor proporción (No Consume), existen 116.041,04 y 145.248,79 veces más de probabilidades de consumir leche ecológica para las categorías Leche entera y Otro tipo de leche.

En el caso de la variable categórica Razón principal por la cual consumiría leche ecológica (Otra razón), frente a Salud, Sostenibilidad ambiental y Propiedades nutricionales, se tienen 188,91, 78,65 y 853,68 veces más de probabilidad de consumir leche ecológica.

Para interpretar los coeficientes relacionados con la Gastos aproximados mensuales del hogar, se debe usar un mecanismo que permita medir el cambio que ocurre en los logit o en las posibilidades, por cada unidad en la que se incrementan los gastos. Una forma es derivar la función, otro medio es obtener el cociente entre las posibilidades en los gastos y gastos+1. El segundo resulta, en general, más sencillo, y se obtiene aquí una función creciente en los gastos.

En la tabla 5 se encuentra que el modelo clasificó bien (97 \%) al comparar lo observado con lo pronosticado: de $106+2$ hogares que no están dispuestos a pagar el $98 \%$ no están dispuestos en el pronóstico; de 189+7 hogares dispuestos a pagar el $96,4 \%$ está pronosticado dispuesto.

\footnotetext{
12 Para interpretar los resultados se advierte que los niveles de referencia para la variable cualitativa $\mathrm{P}_{-}$4(1) es si No ha oído hablar de productos ecológicos, para P_10_a y P_11_a si No consume leche y Ningún tipo de leche, y para P_ 16a Otra razón por la cual consumiría leche ecológica.
} 
Alejandra Calderón García - David Tobón Orozco - Valentina Cardona Nieto - Gabriel Agudelo Viana

Tabla 5: Clasificación disponibilidad a pagar por leche ecológica entera (1)

\begin{tabular}{|c|c|c|c|c|c|}
\hline & & & \multicolumn{3}{|c|}{ Pronosticado } \\
\hline \multirow{2}{*}{\multicolumn{3}{|c|}{ Observado }} & \multicolumn{2}{|c|}{$\begin{array}{c}\text { P.14.E ¿Estaría dispuesto a pagar } \\
\text { por la leche ecológica entera? }\end{array}$} & \multirow{2}{*}{ Porcentaje correcto } \\
\hline & & & 0. No & l.Si & \\
\hline \multirow{3}{*}{ Paso 1} & \multirow{2}{*}{$\begin{array}{c}\text { P.14.E ¿Estaría dispuesto a pagar } \\
\text { por la leche ecológica entera? }\end{array}$} & 0 . No & 106 & 2 & 98,1 \\
\hline & & 1.Si & 7 & 189 & 96,4 \\
\hline & \multicolumn{2}{|l|}{ Porcentaje global } & & & 97,0 \\
\hline
\end{tabular}

Fuente: elaboración propia

En el anexo 2 se incluyen las pruebas de significancia, ajuste y robustez de los modelos, estas son las de Omnibus, Hosmer y Lemeshow (H-L), R², Cox y Snell, y el gráfico de la distribución logística estimada.

En el modelo dos se tomó como variable dependiente la disponibilidad a pagar por leche deslactosada ecológica, pero solo se incluyeron las variables significativas: cantidad de litros de leche consumida en el hogar semanales ( $\mathrm{P}_{-} 10_{-}$a), tipo de leche comprada en mayor proporción ( $\mathrm{P}_{-}{ }^{1} 1_{-}$a) y lo máximo que estarían dispuestos a pagar ( $\mathrm{P}_{\text {_ }} 14$ _ Total _ Máx).

Tabla 6: Variables modelo de regresión leche ecológica deslactosada (2)

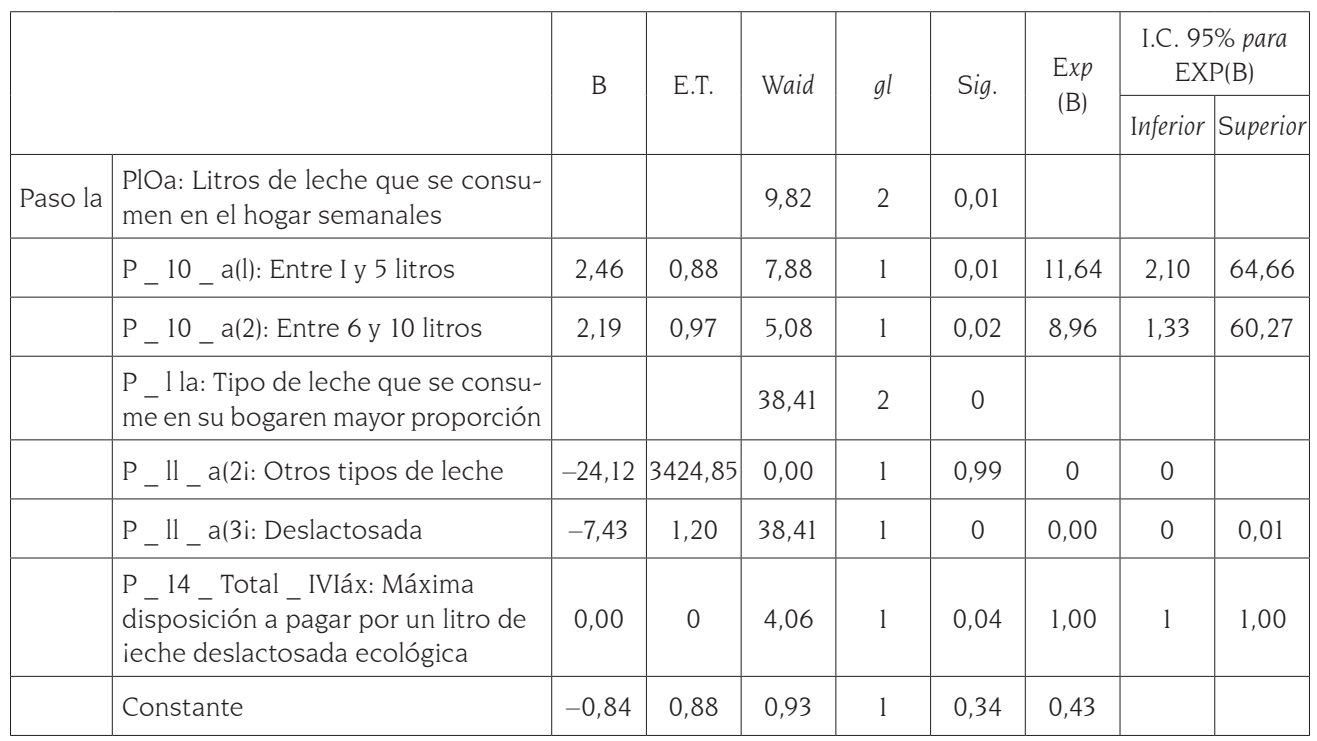

a. Variable(s) introducida(s) en el paso 1: P 10 a, P 11 a, P 14 Total Max.

Fuente: elaboración propia 
La columna del test de Wald (tabla 6) confirma que las variables son significativas con un p-valor $<0,05$, excepto otros tipos de leche. Los coeficientes B muestran que cuando son positivos se incrementa la probabilidad de disponibilidad a pagar por leche ecológica deslactosada, sobre todo en los que consumen entre 1-5 litros. Al interpretar el coeficiente en términos de un efecto multiplicativo, por un incremento de una unidad en los que toman entre 1-5 y 6-10 litros a la semana hay un alto efecto en la razón de probabilidad $(11,643$ y 8,96), en comparación con la variable máxima disponibilidad a pagar $(1,00)$. El tipo de leche consumida no tiene impacto marginal positivo en la probabilidad.

En la tabla 7 se observa que el modelo clasificó bien (97 \%): de 198+8 hogares que no están dispuestos a pagar por un litro de leche deslactosada el 96,1 \% se prevé como no dispuestas. Asimismo, discriminó que de 1+97 hogares dispuestos a pagar el $99 \%$ se estima como dispuestos.

Tabla 7: Clasificación disponibilidad a pagar por leche ecológica deslactosada (2)

\begin{tabular}{|c|c|c|c|c|c|}
\hline & & & & onosticado & \\
\hline & Observado & & $\begin{array}{l}\text { P.14.D ¿E } \\
\text { por la lech }\end{array}$ & $\begin{array}{l}\text { to a pagar } \\
\text { latosada? }\end{array}$ & Porcentaje correcto \\
\hline & & & 0 . No & l.Si & \\
\hline & P.14.D ¿Estaría dispuesto a pagar & 0. No & 198 & 8 & 96,1 \\
\hline Paso 1 & por la leche ecológica deslactosada? & 1. Si & 1 & 97 & 99 \\
\hline & Porcentaje global & & & & 97 \\
\hline
\end{tabular}

El valor de corte es 0,50

Fuente: elaboración propia

Por último, siguiendo a Valencia, Tobón y Becerra (2007), como se han modelado distribuciones logísticas para los errores de estimación $\eta$, luego de encontrados los estimados $\hat{\alpha}$ y $\hat{\beta}$, se puede predecir la DAP. Teniendo que $\eta=\hat{\alpha}, S-\hat{\beta} A, \hat{\alpha}$, es el vector de coeficientes asociados al conocimiento del producto y el consumo de leche, y $\hat{\beta}$, la utilidad marginal del gasto, entonces $A=\hat{\alpha} \cdot S / \beta$. Se estimó que los consumidores están dispuestos a pagar hasta $\$ 3.112$ pesos por un litro de leche entera (primer modelo) y \$3.791 pesos por la leche ecológica deslactosada.

\section{CONCLUSIONES}

La lechería ecológica es una alternativa para reducir la contaminación provocada en sistemas de producción tradicionales y mejorar las calidades nutricionales de la leche. 
Basados en el ejercicio empírico, puede afirmarse que existe un mercado potencial para la leche ecológica, con unas disponibilidades a pagar promedio en la encuesta de 3720 y 2921 para las leches ecológicas deslactosada y entera, y estimadas de 3791 y 3112. De este mercado potencial hacen parte los consumidores habituales de leche y quienes tienen mayores gastos, y la estrategia de comercialización más adecuada es proveer información sobre las características del producto y los beneficios en salud y nutricionales. Las DAP encontradas permiten compensar los costos requeridos para la conversión y reducción de la cantidad producida de leche.

Este mercado potencial si bien cubriría solo un porcentaje menor de la demanda total de leche, alinearía los incentivos entre las dificultades de aplicar la regulación ambiental al sector y motivar una producción más sostenible.

Los mayores problemas para la reconversión de una ganadería convencional por una ecológica son la falta de disposición de los productores, por su resistencia al cambio, y la poca información sobre estos mercados. Este trabajo ayuda a tener información más precisa sobre los costos de oportunidad de encarar la reconvención. Por último, no se puede ignorar la restricción dada por el monopsonio en la compra de la leche líquida en Colombia por parte de pasteurizadoras como Colanta, Alpina, Alquería, Parmalat, Proleche, y la prohibición que tienen los productores de vender leche cruda, por lo que la introducción de un nuevo producto en el mercado debe contar con la asistencia de estas comercializadoras.

\section{BIBLIOGRAFÍA}

Borregaard, N., Dufey, A., Geisee, G. y Ladrón de Guevara, J. (2003). Mercados verdes. Oportunidades prometedoras y desafiantes. [En línea] Centro de Investigación y Planificación del medio ambiente (CIPMA) y Recursos e investigación para el Desarrollo Sustentable (RIDES), Santiago (Chile), 171 p., <http://www.cipma.cl/images/stories/mercados-verdes-oportunidades-prometedoras-y-desafiantes.pdf $>$. [Noviembre 2012].

Bradford, L., Barham, L., Brock, C., y Foltz, J. (2006). Organic Dairy Farms in Wisconsin: Prosperous, Modern, and Expansive. En: PATS Research Report, N. ${ }^{\circ}$ 16, pp. 1-18.

Brécard, D., Hlaimi, B., Lucas, S., Perraudeau, Y. y Salladarré, F. (2009). Determinants of Demand for Green Products: an application to eco-label demand for fish in Europe. En: Ecological Economics, Vol. 69, N. ${ }^{\circ}$ 1, pp. 115-125.

Carmona, J. Bolívar, D. y Giraldo, L. (2005). El gas metano en la producción ganadera y alternativas para medir sus emisiones y aminorar su impacto a nivel ambiental y productivo. En: Revista Colombiana de Ciencias Pecuarias, Vol. 18, N. 1, pp. 49-63. 
Características, incentivos a la producción y disponibilidad a pagar por leche ecológica en Medellín

Colombia, Alcaldía de Medellín (2011). Encuesta de Calidad de Vida. Perfiles por estrato. [En línea]. Departamento Administrativo de Planeación, 20 p., <http://www.medellin.gov.co/ irj/portal/ciudadanos?NavigationTarget=navurl://3e7291b8190ecb22ebb47c5b0d660f8a>. [Noviembre 2012].

Colombia, Congreso de la República (1993). Ley General Ambiental (Ley 99). Bogotá, 316 pp.

Colombia, MADR -Ministerio de Agricultura y Desarrollo Rural (2012). Resolución 017. Por la cual se establece el sistema de pago de leche cruda al proveedor. Bogotá, 17 pp.

Colombia, MADR - Ministerio de Agricultura y Desarrollo Rural (2006). Reglamento para la producción primaria, procesamiento, empacado, etiquetado, almacenamiento, certificación, importación y comercialización de Productos Agropecuarios Ecológicos. [En línea] 47 pp. < http://www.agriculturaorganicaamericas.net/Documentos\%200ficiales\%20CIAO/ Reglamento.pdf $>$ [Agosto 2014].

Colombia, MADR -Ministerio de Agricultura y Desarrollo Rural (2007). La agricultura ecológica en Colombia. [En línea] 38 pp. < http://www.agronet.gov.co/www/docs _ agronet/2007423154157 _ Articulo _ agricultura\%20ecologica\%202007\%20MADR\%20.pdf $\geq$. [Agosto 2014].

Colombia, MADS -Ministerio de Ambiente y Desarrollo Sostenible (2005). Resolución 1555. Sello ambiental colombiano. Bogotá, 15 pp.

Colombia, MADS -Ministerio de Ambiente y Desarrollo Sostenible (2002). Plan Estratégico Nacional de Mercados Verdes (PENMV). [En línea] 84 pp. https://www.dnp.gov.co/Portals/0/archivos/ documentos/DDUPA/Medio_Ambiente/P\%C3\%Algina6_Plan _ Estrat\%C3\%A9gico_Programa_Mercados_Verdes.PDF. [Noviembre 2012].

Colombia, Presidencia de la República (2012). Decreto 2667. Por el cual se reglamenta la tasa retributiva. Bogotá, 15 pp.

Colombia, Presidencia de la República (2010). Decreto 3930. Por el cual se reglamenta los vertimientos de agua y residuos líquidos. Bogotá, 93 pp.

Colombia, Presidencia de la República (2006). Decreto 616. Reglamento técnico sobre los requisitos que debe cumplir la leche para consumo humano. Bogotá, 40 pp.

Corantioquia -Corporación Autónoma Regional del Centro de Antioquia, Universidad Nacional y Universidad de Antioquia (2012). Valoración económica, ecológica y socio - cultural de bienes y servicios ambientales en la cuenca del Río Grande. Aproximación conceptual y metodológica. Medellín, Mimeo, 560 pp.

EMPRENDEGAN (2010). Aplicativo San José 2010. Estructura de costos Hacienda San José, Bogotá, FEDEGAN, 6 pp. 
Espinal, C., Martínez, J. y Espinosa, D. (2005). La cadena de cultivos ecológicos en Colombia Una mirada global de su estructura y dinámica 1991-2005. [En línea] Observatorio agrocadenas Colombia, Ministerio de Agricultura y Desarrollo Rural. Documento de trabajo N. ${ }^{\circ}$ 68, pp. 1-28, < http://www.agronet.gov.co/www/docs _ agronet/20051121601 _ caracterizacion_ecologicos.pdf $>$. [Junio 2012].

FEDEGAN -Federación Colombiana de Ganaderos (2011). Hacienda San José - Juan Guillermo Luna. En la onda de lo orgánico. En: Carta FEDEGAN, N.1124, pp. 70-72.

Fernández, H. y Pérez, F. (2005). El modelo logístico: Una herramienta estadística para evaluar el riesgo de crédito. En: Revista de Ingenierías Universidad de Medellín, Vol. 4, N. ․ 6p. pp. 55-75.

Frey, B. (1999). Morality and Rationality in Environmental Policy. En: Journal of Consumer Policy, Vol. 4, N. ${ }^{\circ}$, December 1999, Volume 22, Issue 4, pp. 395-417.

Galarza, Miguel Ángel (2009). Análisis de la efectividad de las tasas retributivas en Colombia. Estudio de caso. Tesis para optar al título de economista, Facultad de Ciencias Administrativas y Económicas, Pontificia Universidad Javeriana, Bogotá, pp. 49-75.

Mahecha, Liliana (2002). El silvopastoreo: una alternativa de producción que disminuye el impacto ambiental de la ganadería bovina. En: Revista Colombiana Ciencias Pecuarias, Vol. $15,{ }^{\circ}{ }^{\circ}$, pp. 226-231.

Mahenc, Philippe (2008). Introducing Greens Goods. En: LERNA Working Papers 08.03.247, Toulouse, LERNA, University of Toulouse, $38 \mathrm{pp}$.

Márquez, Sara (2011). Evaluación de los riesgos ambientales por la aplicación de clorpirifos en zonas de ganadería de leche, en el municipio de San Pedro de los Milagros. Tesis Doctoral, Universidad de Antioquia. Mei, O. J., Ling K. C. y Hooi K. K. (2012). The Antecedents of Green Purchase Intention among Malaysian Consumers. [En línea] International Proceedings of Economics Development and Research [IPEDR], Vol. 38, art. 8, pp. 39-43 http://www.ipedr. com/vol38/008-ICEBI2012-A00014.pdf. [Enero 2013].

Mena, Leticia (2009). Leche ecológica. [En línea] El diario montañés, http://www.eldiariomontanes. es/20090620/sociedad/cantabria-mesa/leche-ecologica-20090620.html. [Noviembre 2012].

Murgueitio, Enrique (1999). Reconversión ambiental y social de la ganadería bovina en Colombia. En: Revista Mundial de Zootecnia, Vol. 93, N.․ 2, pp. 2-15.

Navia, J. (2000). Agroforestería. [En línea] Centro para el desarrollo agropecuario y forestal (CEDAF), 182 p. <http://www.cedaf.org.do/CENTRODOC/EBOOK/AGROFORESTERIA.PDF>. [Agosto 2014].

Observatorio de competitividad Corporación Colombiana Internacional (2002). Mercado Mundial de ecológicos con énfasis en cacao, panela, banano y frutas promisorias. [En línea] 29 p. <http://www.panelamonitor.org/documents/165/mercado-mundial-de-ecologicos-con-enfasis-en-cacao/> [Enero 2013]. 
Características, incentivos a la producción y disponibilidad a pagar por leche ecológica en Medellín

Osorio, J. D. y Correa, F. (2004). Valoración económica de costos ambientales: Marco conceptual y métodos de estimación. En: Revista Semestre Económico, Vol. 7, N.1․ 13p. 159-193.

Pérez, J. A. y Álvarez, A. (2008). Análisis económico de la producción de leche ecológica. En: Tribuna de Economía, Revista ICE, No 843, pp. 227-240.

Schröck, Rebecca (2011). The Organic Milk Market in Germany is Maturing: A Demand System Analysis of Organic and Conventional Fresh Milk in Germany Segmented by Consumer Groups. [En línea] The European Association of Agricultural Economists (EAAE), 12 p. $<$ http://ageconsearch.umn.edu/bitstream/115995/2/Schroeck_ Rebecca _ 186.pdf>. [Enero 2013].

Tanner, C. y Wolfing, S. (2003). Promoting Sustainable Consumption: Determinants of Green Purchases by Swiss Consumers. En: Psychology \& Marketing, Vol. 20, N. 10, pp. 27-39.

Tobón, D. y Vasco, C. (2011). Modelo de equilibrio general con externalidades y capital natural. Medellín, Centro de Investigaciones y Consultorías -CIC-, Universidad de Antioquia, 98 pp.

Toro, M. y Madrid, J. (2012). Producción de leche orgánica. Una forma de agregar valor desde la finca. En: Revista COLANTA Pecuaria. Revista para el sector lácteo y cárnico, Edición 33, pp. 49-57.

Valencia, G., Tobón, D. y Becerra, Y. (2007). Pérdida de bienestar en los pasajeros del transporte aéreo: el caso Alianza Summa en la ruta Medellín-Bogotá. En: Revista Cuadernos de Administración, Vol. 20, N. ${ }^{\circ} 33$, pp. 147-179.

Van Hoof, B., Monroy, N. y Saer, A. (2008). Producción más limpia. Paradigma de gestión ambiental, cap. 3, Bogotá, Colombia: Alfaomega Grupo Editor y Universidad de los Andes, pp. 1-23.

Xia, W. y Zeng, Y. (2009). Consumer's attitudes and willingness-to-pay for Green food in Beijing. En: Food Control, Vol. 33, N.1, pp. 93-104.

YR, A. (2011). Sistemas agroforestales como alternativa de manejo sostenible en la actividad ganadera de la Orinoquia Colombiana. En: Revista Sistemas de Producción Agroecológicos, Vol. 2, N. ${ }^{\circ}$, pp. 103-127.

\section{ENTREVISTAS}

Márquez, Sara (2012). Docente, Facultad de Ciencias Agrarias de la Universidad de Antioquia. Medellín, 1 de agosto.

Martínez, Héctor (2012). Productor finca lechera en San Pedro de los Milagros (Antioquia). San Pedro de los Milagros, 12 de noviembre.

Ospina, Humberto (2012). Productor finca lechera en San Pedro de los Milagros (Antioquia). San Pedro de los Milagros, 12 de noviembre. 
Alejandra Calderón García - David Tobón Orozco - Valentina Cardona Nieto - Gabriel Agudelo Viana

Velásquez, Gildardo (2012). Administrador finca productora de la familia García. San Pedro de los Milagros (Antioquia), 10 de octubre. 


\section{ANEXO A}

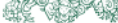

UNIVERSIDAD

\section{ENCUESTA SOBRE DISPONIBILIDAD A PAGAR POR LA LECHE ECOLÓGICA}

Fecha: Día

Mes:

Hora Inicio:

Hora Finalización:

Lugar: 1 .

2.

3.

Nombre encuestador:

Sexo del encuestado: $\mathrm{F}$ M

¿Es usted jefe del hogar?

¿Consumen leche en su hogar?

\section{PRESENTACIÓN}

Se realiza un estudio para obtener la valoración económica de un nuevo producto en Medellín ("leche ecológica"), con importantes características para la Salud y el Ambiente. El cuestionario es voluntario y la información suministrada será utilizada para fines académicos. Le solicitamos responda con la mayor sinceridad.

La encuesta aplica a personas mayores de 18 años y a cargo de las compras del hogar, dura solo 15 minutos.

\section{Módulo de percepción}

P.1 En una escala de 1 a 5 donde 1 significa poco y 5 mucho, clasifique en qué grado realiza actividades que ayuden al cuidado y conservación del ambiente.
P.2 ¿Cuál de los siguientes temas de interés general es el más importante para Ud.? (Solo una opción).

1. Empleo

2. Seguridad

3. Sostenibilidad Ambiental

4. Educación

5. Recreación

6. Salud

P.4 ¿Ha oído hablar de los productos ecológicos?

Sí__ NO _ _
P.3 ¿Cree que los productos lácteos que consume provocan afectaciones al ambiente?

sí__ NO 


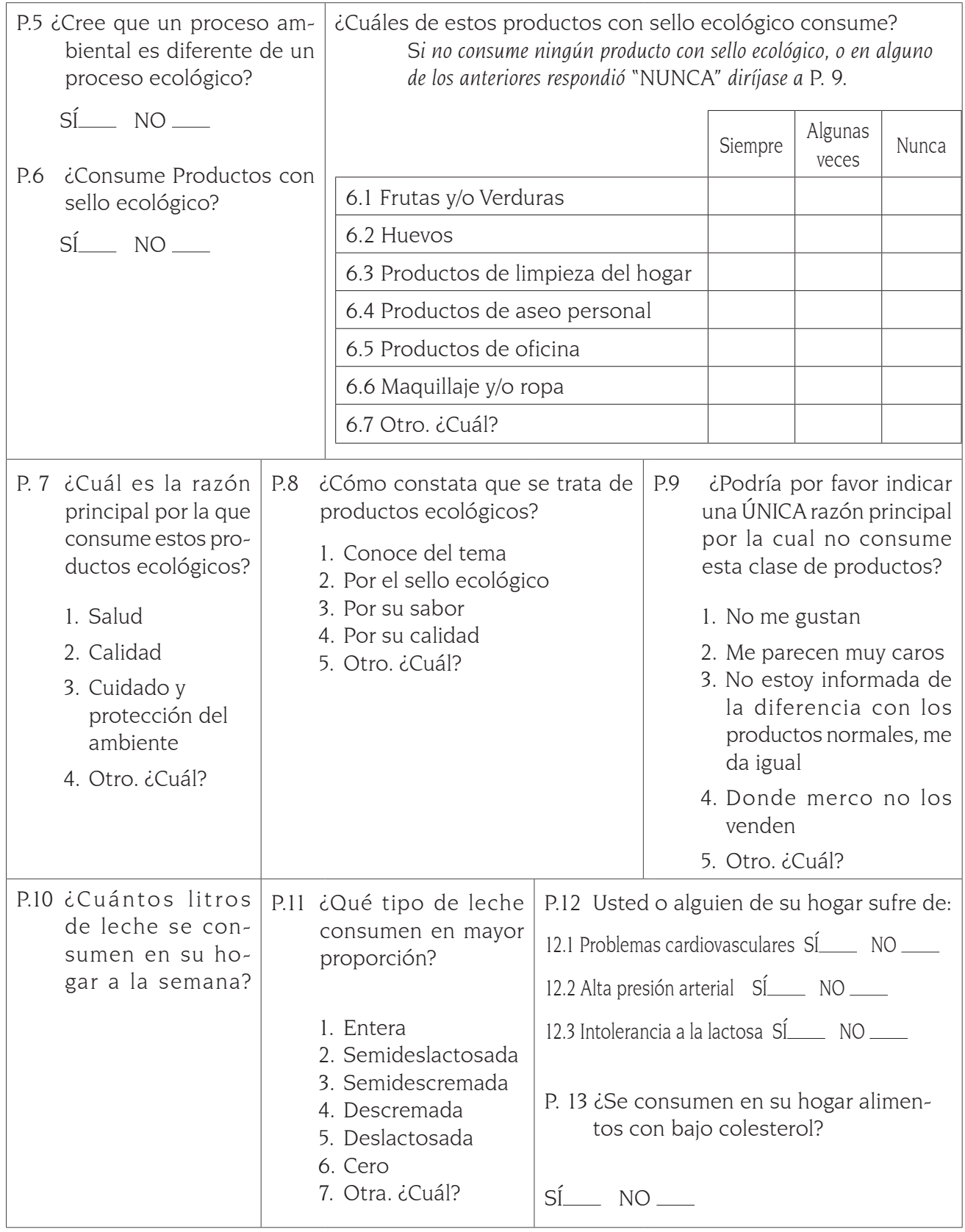

Información: Se informa al encuestado sobre las características de la producción de leche convencional y leche ecológica, y que en Colombia no se vende esta última.

Se realizó un solo formato de encuesta, se mencionan cuatro tipos de encuesta que solo difieren en la puja inicial del juego iterativo de preguntas de disponibilidad a pagar por leche ecológica y deslactosada. 


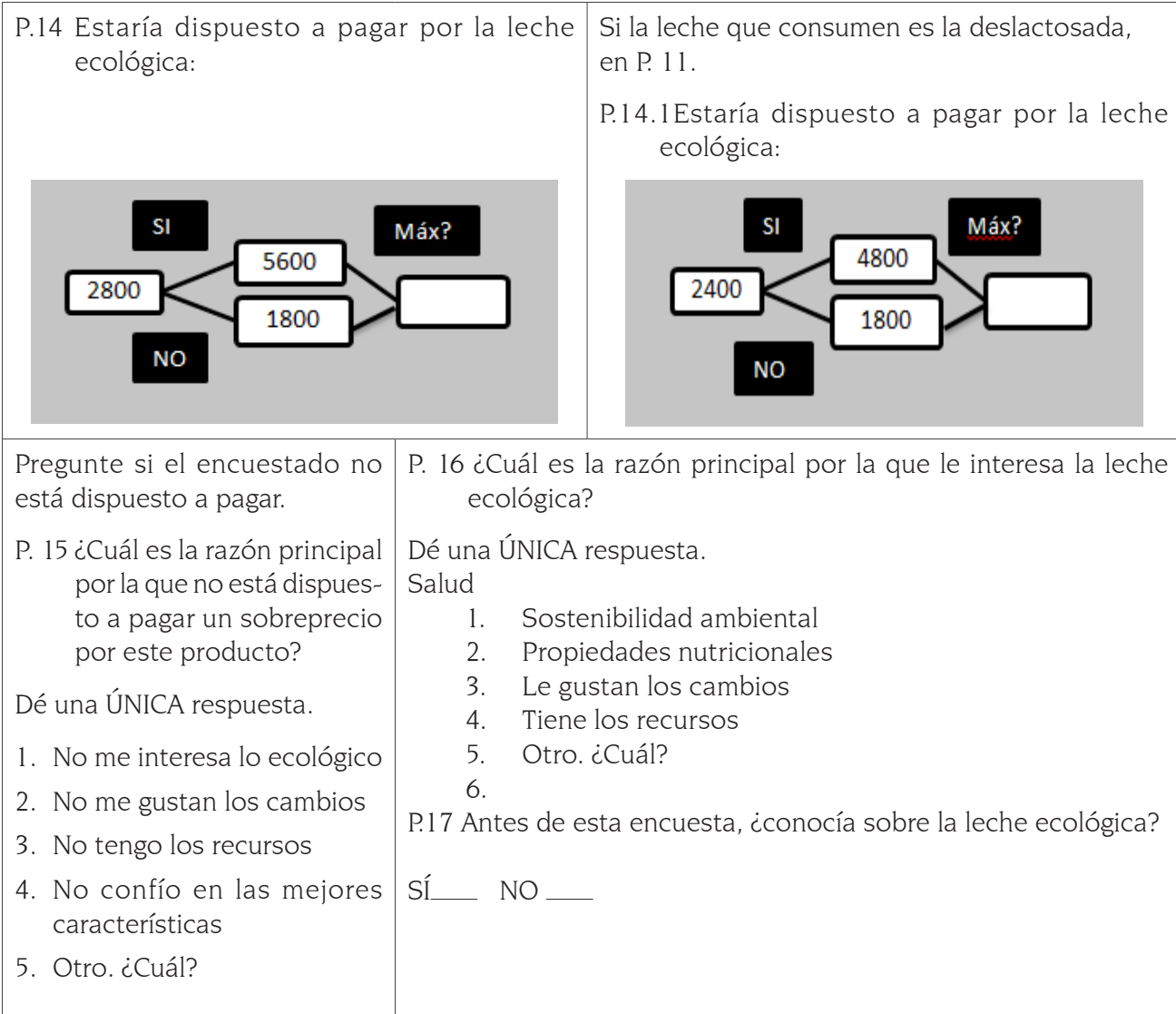

\section{Módulo de características sociodemográficas}

P. 1 Año de nacimiento:

(Edad estimada por el encuestador )

P. 2 Estado civil:

Soltero (a)

Casado (a)

Viudo (a)

Divorciado (a)

Separado (a)

Unión libre

P. 3 Estrato indicado en el recibo de servicios públicos domiciliarios

$1 \_2-3-4-5-6$




\begin{tabular}{|c|c|}
\hline $\begin{array}{l}\text { P.4 Último nivel de estudio: } \\
\begin{array}{ll}\text { 1. Ninguno } \\
\text { 2. Preescolar } \\
\text { 3. Primaria } \\
\text { 4. Secundaria } \\
\text { 5. Técnico } \\
\text { 6. Tecnológico } \\
\text { 7. Universitario } \\
\text { 8. Posgrado } \\
\text { 9. Otro. (¿Cuál?) }\end{array}\end{array}$ & $\begin{array}{l}\text { P. } 5 \text { Actualmente usted es: } \\
\text { 1. Empresario } \\
\text { 2. Empleado doméstico } \\
\text { 3. Trabajador empleado } \\
\text { 4. Pensionado } \\
\text { 5. Desempleado } \\
\text { 6. Vendedor ambulante } \\
\text { 7. Rentista (vive de arriendos) } \\
\text { 8. Estudiante } \\
\text { 9. Ama de casa } \\
\text { 10. Trabajador independiente } \\
\text { 11. Otro. (¿Cuál?) }\end{array}$ \\
\hline $\begin{array}{l}\text { P. } 7 \text { Ubique los ingresos mensuales en alguno de } \\
\text { los siguientes rangos: } \\
\text { Rango de Ingresos mensuales en SMMLV } \\
\text { 1. Entre } 1-3 \text { SMMLV (566 y 1'700) } \\
\text { 2. Entre } 3.1-5 \text { SMMLV }\left(1^{\prime} 701-2^{\prime} 830\right) \\
\text { 3. Entre } 5.1-10 \text { SMMLV (2'831-5'600) } \\
\text { 4. Más de } 10.1 \text { SMMV (más de 5'601) }\end{array}$ & P. 8 ¿Cuántas personas dependen de su ingreso? \\
\hline 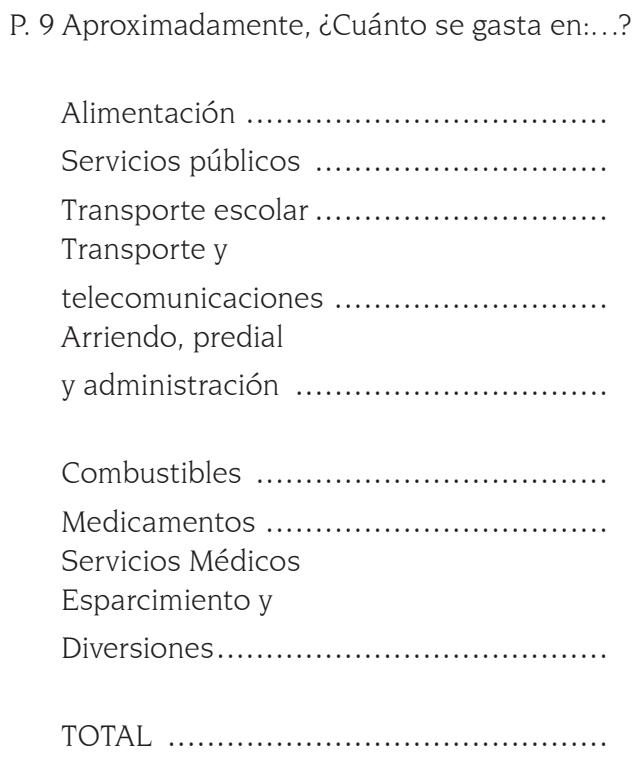 & $\begin{array}{l}\text { P.10 Incluido usted. ¿Cuántas personas confor- } \\
\text { man su hogar? } \\
\text { 10.1 ¿Cuántos niños? } \\
\text { De } 0-12 \text { años } \\
\text { 10.2 ¿Cuántos adolescentes? } \\
\text { De 13-18 años } \\
\text { P.11 ¿Cuántas personas NO consumen leche en } \\
\text { su hogar? }\end{array}$ \\
\hline
\end{tabular}




\section{ANEXO B}

\section{Modelo 1}

La prueba Omnibus analiza la significancia de las componentes conjuntas del modelo, donde: $\mathrm{H}_{0}: \beta_{1}=\beta_{2}=\ldots=\beta_{6}=0$ vs. $\mathrm{H}_{1}$ : Al menos un parámetro diferente de cero. Como el valor $\rho<0.05$, se rechaza la hipótesis nula, y al menos una de las componentes es significativa.

Para la bondad del ajuste se usa la prueba de Hosmer y Lemeshow (H-L): $\mathrm{H}_{0}$ : El modelo está bien ajustado frente a $\mathrm{H}_{1}$ : Falta ajuste en el modelo. El valor de $\mathrm{H}$-L: 6,60 indica una buena calibración; además, el valor $p>0,05$, lo que lleva a aceptar la hipótesis nula. Lo que busca la prueba es que no haya significación, es decir, mientras más cercano a 1 esté el p-valor mejor el resultado. Pero al tener en cuenta las posibles inconsistencias, dado que la prueba no computa los grupos de valores esperados que son nulos o muy pequeños, se observa la tabla de contingencias en la cual el modelo tiene un buen ajuste, ya que la cantidad de datos observados coincide con los esperados, tanto para los que están dispuestos a pagar como para los que no están dispuestos (tabla 8).

Tabla 8: Resultados prueba Hosmer-Lemeshow (1)

\begin{tabular}{|c|c|c|c|c|c|c|}
\hline \multirow{2}{*}{\multicolumn{2}{|c|}{ Observado }} & \multicolumn{2}{|c|}{$\begin{array}{l}\text { P.14.E ¿Estaría dispuesto a } \\
\text { pagar por la leche ecológica } \\
\text { entera? }=0 \text {. No }\end{array}$} & \multicolumn{2}{|c|}{$\begin{array}{l}\text { P.14.E ¿Estaría dispuesto a } \\
\text { pagar por la leche ecológica } \\
\text { entera? }=1 . \mathrm{Si}\end{array}$} & \multirow{3}{*}{$\begin{array}{r}\text { Tota } \\
33\end{array}$} \\
\hline & & \multirow{2}{*}{$\begin{array}{r}\text { Esperado } \\
33\end{array}$} & \multirow{2}{*}{$\frac{\text { Observado }}{32,96}$} & \multirow{2}{*}{$\begin{array}{r}\text { Esperado } \\
0\end{array}$} & \multirow[b]{2}{*}{0,05} & \\
\hline \multirow{10}{*}{ Paso 1} & 1 & & & & & \\
\hline & 2 & 28 & 27,69 & 0 & 0,31 & 28 \\
\hline & 3 & 28 & 28,91 & 3 & 2,09 & 31 \\
\hline & 4 & 17 & 16,82 & 13 & 13,18 & 30 \\
\hline & 5 & 1 & 1,42 & 29 & 28,58 & 30 \\
\hline & 6 & 1 & 0,13 & 29 & 29,87 & 30 \\
\hline & 7 & 0 & 0,05 & 30 & 29,96 & 30 \\
\hline & 8 & 0 & 0,02 & 29 & 28,98 & 29 \\
\hline & 9 & 0 & 0,01 & 30 & 29,99 & 30 \\
\hline & 10 & 0 & 0,00 & 33 & 33,00 & 33 \\
\hline
\end{tabular}

Fuente: elaboración propia 
En la tabla 9 se observa que el coeficiente $\mathrm{R}^{2}$ de Nagelkerke es 0,913: al estar bastante cercano a uno significa que la variable dependiente sí está explicada por las independientes incluidas. El coeficiente de determinante de Cox y Snell se usa para estimar la proporción de varianza de la variable dependiente explicada por las variables independientes, el cual es de $66,5 \%$.

Tabla 9: Resumen modelo (1)

\begin{tabular}{|c|c|c|c|}
\hline Paso & $\begin{array}{c}-2 \text { log de la } \\
\text { verosimilitud }\end{array}$ & $\begin{array}{c}\text { R cuadrado de Cox } \\
y \text { Snell }\end{array}$ & $\begin{array}{c}\text { R cuadrado de } \\
\text { Nageikerke }\end{array}$ \\
\hline 1 & $63,36^{\mathrm{a}}$ & 0,67 & 0,91 \\
\hline
\end{tabular}

a: La estimación finaliza en la iteración 9, las estimaciones han cambiado en menos de ,001.

Fuente: elaboración propia

El gráfico 2 corresponde al de distribución logística, en la cual probabilidad pronosticada de la leche entera se expresa en función del score del modelo logístico, es decir, de la valoración que tienen los hogares para determinar si están dispuestos a pagar o no; la relación entre las dos variables describe la forma sigmoidea prevista.

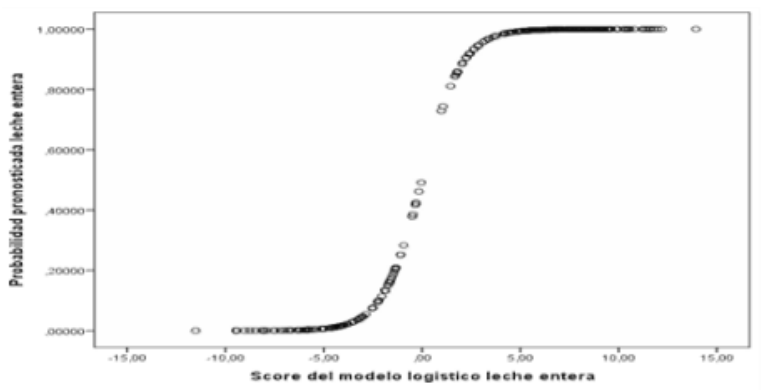

Gráfico 2. Score modelo logístico leche entera ecológica (1)

Fuente: elaboración propia

\section{Modelo 2}

La prueba omnibus arroja un valor $\rho<0,05$, es decir, se rechaza la hipótesis nula y al menos una de las componentes es significativa. Respecto a la prueba $\mathrm{H}-\mathrm{L}$, el Chi cuadrado es de 4,185 y la significancia muy cercana a uno, lo cual indica un buen ajuste. 
Características, incentivos a la producción y disponibilidad a pagar por leche ecológica en Medellín

Tabla 10: Resultados prueba Hosmer-Lemeshow (2)

\begin{tabular}{|c|c|c|c|c|c|c|}
\hline \multirow{2}{*}{\multicolumn{2}{|c|}{ Observado }} & \multicolumn{2}{|c|}{$\begin{array}{l}\text { P.14.D ¿Estaría dispuesto a } \\
\text { pagar por la leche ecológi- } \\
\text { ca deslactosada } ?=0 . \text { No }\end{array}$} & \multicolumn{2}{|c|}{$\begin{array}{l}\text { P.14.D ¿Estaría dispuesto a } \\
\text { pagar por la leche ecológi- } \\
\text { ca deslactosada? }=1 . \mathrm{Si}\end{array}$} & \multirow{3}{*}{$\begin{array}{c}\text { Total } \\
30\end{array}$} \\
\hline & & \multirow{2}{*}{$\frac{\text { Esperado }}{30}$} & \multirow{2}{*}{$\begin{array}{c}\text { Observado } \\
30 \\
\end{array}$} & \multirow{2}{*}{$\begin{array}{c}\text { Esperado } \\
0\end{array}$} & \multirow[b]{2}{*}{0} & \\
\hline \multirow{10}{*}{ Paso 1} & 1 & & & & & \\
\hline & 2 & 30 & 30 & 0 & 0 & 30 \\
\hline & 3 & 29 & 29 & 0 & 0 & 29 \\
\hline & 4 & 31 & 31 & 0 & 0 & 31 \\
\hline & 5 & 27 & 26,93 & 0 & 0,07 & 27 \\
\hline & 6 & 28 & 28,67 & 1 & 0,33 & 29 \\
\hline & 7 & 23 & 23,52 & 5 & 4,49 & 28 \\
\hline & 8 & 7 & 4,84 & 22 & 24,16 & 29 \\
\hline & 9 & 0 & 1,33 & 31 & 29,67 & 31 \\
\hline & 10 & 1 & 0,72 & 39 & 39,29 & 40 \\
\hline
\end{tabular}

Fuente: elaboración propia

Asimismo, los contrastes entre los datos observados y esperados son muy cercanos. El $R^{2}=0,91$, de nuevo cercano a 1. El coeficiente de Cox y Snell muestra que hay una variación del $65 \%$ en la variable dependiente debido a un cambio en las independientes. Y el -2 log de la verosimilitud dice que mientras menor sea este indicador, mayor es la verosimilitud del modelo. Por tanto se presenta una buena verosimilitud ya que el número no es muy grande.

Tabla 11: Resumen modelo (2)

\begin{tabular}{|c|c|c|c|}
\hline Paso & $\begin{array}{c}-2 \text { log de la } \\
\text { verosimilitud }\end{array}$ & $\begin{array}{c}\text { R cuadrado de Cox } \\
y \text { Snell }\end{array}$ & $\begin{array}{c}\text { R cuadrado de } \\
\text { Nageikerke }\end{array}$ \\
\hline 1 & $62,06^{\mathrm{a}}$ & 0,65 & 0,91 \\
\hline
\end{tabular}

a: La estimación ha finalizado en el máximo de iteraciones (20). No hay solución definitiva.

Fuente: elaboración propia

Por último, la representación de la probabilidad de que los encuestados consuman leche deslactosada ecológica en contraste con el score que ofrece el modelo logístico es la esperada (gráfico 3). 
Alejandra Calderón García - David Tobón Orozco - Valentina Cardona Nieto - Gabriel Agudelo Viana

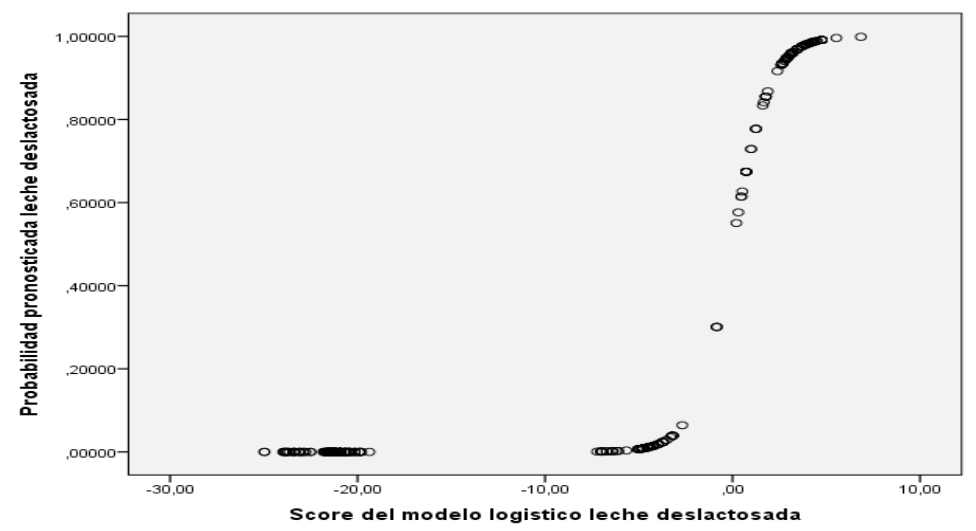

Gráfico 3: Score modelo logístico leche deslactosada ecológica (2)

Fuente: elaboración propia. 